\title{
EXISTENCE, UNIQUENESS, AND SPECTRAL PROPERTIES OF NONLINEAR EIGENVALUE PROBLEMS ${ }^{1}$
}

\author{
DONALD S. COHEN
}

We consider the following nonlinear eigenvalue problem:

$$
\begin{aligned}
\left(p(x) u^{\prime}\right)^{\prime}+\lambda f(x, u) & =0, & 0 \leqq x \leqq 1, & \\
a_{0} u(0)-a_{1} u^{\prime}(0) & =0, & & \left|a_{0}\right|+\left|a_{1}\right| \neq 0, \\
b_{0} u(1)+b_{1} u^{\prime}(1) & =0, & & \left|b_{0}\right|+\left|b_{1}\right| \neq 0 .
\end{aligned}
$$

We suppose that $p(x)>0$ and $p^{\prime}(x)$ is continuous on $0 \leqq x \leqq 1$ and that $f(x, u)$ satisfies the following conditions:

H-1: $f(x, u)$ is continuously differentiable in $D$ :

$$
0 \leqq x \leqq 1, \quad-\infty<u<\infty \text {. }
$$

H-2: $0<f_{u}(x, u)<\rho(x)$ on $D$, where $\rho(x)>0$ in $0 \leqq x \leqq 1$.

$\mathrm{H}-3: f(x, 0) \not \equiv 0$ on $0 \leqq x \leqq 1$.

Our main result is the

Theorem. Let $f(x, u)$ satisfy $\mathrm{H}-1,2,3$, and let the constants $a_{i}, b_{i}$ satisfy

$$
a_{i} \geqq 0, \quad b_{i} \geqq 0, \quad(i=0,1), a_{0}+b_{0}>0 .
$$

Then, there exists a unique solution of (1), (2), (3) for all $\lambda$ in $0<\lambda$ $<\mu_{1}\{\rho\}$, where $\mu_{1}\{\rho\}$ is the principal (i.e., least) eigenvalue of

$$
\begin{aligned}
\left(p(x) u^{\prime}\right)^{\prime}+\mu \rho(x) u & =0, \quad 0 \leqq x \leqq 1, \\
a_{0} u(0)-a_{1} u^{\prime}(0) & =0, \\
b_{0} u(1)+b_{1} u^{\prime}(1) & =0 .
\end{aligned}
$$

Proof. We outline the proof which is based on a technique used recently by $H$. B. Keller [1]. The initial value problem

$$
\begin{aligned}
\left(p(x) y^{\prime}\right)^{\prime}+\lambda f(x, y) & =0, \\
a_{0} y(0)-a_{1} y^{\prime}(0) & =0, \\
c_{0} y(0)-c_{1} y^{\prime}(0) & =s, \quad a_{1} c_{0}-a_{0} c_{1}=1,
\end{aligned}
$$

has the unique solution $y(s ; x)$. The problem (1), (2), (3) has as many solutions as there are real roots, $s^{*}$, of

Received by the editors October 7, 1966.

1 This work was supported by the National Science Foundation under Grant No. GP-4597 at the California Institute of Technology. 


$$
\phi(s) \equiv b_{0} y(s ; 1)+b_{1} y^{\prime}(s ; 1)=0 .
$$

We shall show that $\phi^{\prime}(s)$ is positive and bounded away from zero, from which it follows that $\phi(s)=0$ always has one and only one root.

Since $y(s ; x)$ is continuously differentiable with respect to $s$, the derivative $w(x) \equiv \partial y(s ; x) / \partial s$ satisfies the variational problem

$$
\begin{aligned}
\left(p(x) w^{\prime}\right)^{\prime}+\lambda f_{u}(x, y) w & =0, \\
a_{0} w(0)-a_{1} w^{\prime}(0) & =0, \\
c_{0} w(0)-c_{1} w^{\prime}(0) & =1 .
\end{aligned}
$$

Clearly we must show that $\phi^{\prime}(s) \equiv b_{0} w(1)+b_{1} w^{\prime}(1)$ is positive and bounded away from zero. To do this we consider the linear problem

$$
\begin{aligned}
\left(p(x) v^{\prime}\right)^{\prime}+\lambda \rho(x) v & =0, \\
a_{0} v(0)-a_{1} v^{\prime}(0) & =0, \\
c_{0} v(0)-c_{1} v^{\prime}(0) & =1 .
\end{aligned}
$$

For a fixed $\lambda \equiv \lambda_{1}$, say, let $l$ be the first value of $x>0$ at which $b_{0} v(l)$ $+b_{1} v^{\prime}(l)=0$. (That such an $l$ exists will be clear from the formulation of problem (13), (14), (15).) Then, the unique solution $v_{1}(x)$ of (10), (11), (12) also satisfies

$$
\begin{aligned}
\left(p(x) v_{1}^{\prime}\right)^{\prime}+\lambda_{1} \rho(x) v_{1} & =0, \\
a_{0} v_{1}(0)-a_{1} v_{1}^{\prime}(0) & =0, \\
b_{0} v_{1}(l)+b_{1} v_{1}^{\prime}(l) & =0 .
\end{aligned}
$$

where $\lambda_{1}=\lambda_{1}(l)$ is the principal eigenvalue of (13), (14), (15) and $v_{1}(x)$ is the corresponding eigenfunction normalized so that it satisfies (12).

We now show that $b_{0} w(x)+b_{1} w^{\prime}(x)>0$ on $0<x<l$. We do this by contradiction. If $b_{0} w(\kappa)+b_{1} w^{\prime}(\kappa)=0$ for some $\kappa$ in $0<\kappa<l$, then $w(x)$ would satisfy

$$
\begin{aligned}
\left(p(x) w^{\prime}\right)^{\prime}+\lambda_{1} f_{u}(x, y) w & =0, \\
a_{0} w(0)-a_{1} w^{\prime}(0) & =0, \\
b_{0} w(\kappa)+b_{1} w^{\prime}(\kappa) & =0 .
\end{aligned}
$$

Now, from the usual variational characterization [2] of the principal eigenvalue of problems of the form of (13), (14), (15), we know that as the coefficient $\rho(x)$ varies in one sense, the eigenvalue $\lambda_{1}$ varies in the opposite sense, and as the length of the interval varies in one sense, the eigenvalue $\lambda_{1}$ varies in the opposite sense. Thus, for fixed $\lambda=\lambda_{1}$, since $f_{u}(x, y)<\rho(x)$, equation (18) can not hold for $\kappa<l$. Hence, we conclude that $b_{0} w(x)+b_{1} w^{\prime}(x)>0$ on $0<x<l$. 
Finally, by once again using the fact that $\lambda_{1}(l)$ varies in the opposite sense from $l$, we conclude that if $\lambda<\lambda_{1} \equiv \mu_{1}\{\rho\}$, then $l>1$. Therefore, $\phi^{\prime}(s) \equiv b_{0} w(1)+b_{1} w^{\prime}(1)>0$. Q.E.D.

REMark. Actually, condition H-3 is not necessary for our proof. However, if $f(x, 0) \equiv 0$, the unique solution will be the trivial one. If $f(x, 0)=0$, then the problem is closely related to one treated thoroughly by G. H. Pimbley [3]. Pimbley's Theorem 1 gives uniqueness in the same range of $\lambda$. The extension to the case $f(x, 0) \neq 0$ is by no means trivial, however, and the consequences of this condition are pointed out in some detail in [4].

Recently, H. B. Keller and the present author [4] studied eigenvalue problems of a more general nature than (1), (2), (3) with regard to finding those values of $\lambda$ for which the problem has positive solutions, $u(x)>0$. Such problems arise in the theory of nonlinear heat condition where the function $f(x, u)$ is always positive for $u \geqq 0$. We defined the set $\{\lambda\}$ of real values of $\lambda$ for which positive solutions exist as the spectrum of the problem, and the least upper bound of the spectrum was denoted by $\lambda^{*}$. For the problem (1), (2), (3) with $f$ positive and concave we proved that a unique positive solution exists for all $\lambda$ in $0<\lambda<\lambda^{*}$, bounds were given on $\lambda^{*}$, and in the case that $\lim _{\phi \rightarrow \infty}\left[f_{u}(x, \phi)\right]=\rho(x)>0$, we showed that $\lambda^{*}=\mu_{1}\{\rho\}$. Exact solutions of several simple problems involving convex nonlinearities show that more than one positive solution exists for all $\lambda$ in $0<\lambda<\lambda^{*}$. Upon combining the results in [4] with the theorem of the present paper we can show that for positive convex nonlinearities $f(x, u)$ satisfying $\lim _{\phi \rightarrow \infty}\left[f_{u}(x, \phi)\right]=\rho(x)>0$, a unique positive solution of (1), (2), (3) exists for all $\lambda$ in $0<\lambda<\mu_{1}\{\rho\} \leqq \lambda^{*}$. Thus, for $f$ convex a necessary condition for nonuniqueness is that $f_{u}(x, u)$ be unbounded in $u$.

\section{REFERENCES}

1. H. B. Keller, Existence theory for two point boundary value problems, Bull. Amer. Math. Soc. 72 (1966), 728-731.

2. R. Courant and D. Hilbert, Methods of mathematical physics, Vol. I, Interscience, New York, 1953.

3. G. H. Pimbley, A sublinear Sturm Liouville problem, J. Math. Mech. 11 (1962), 121-138.

4. H. B. Keller and D. S. Cohen, Some positone problems in nonlinear heat conduction, J. Math. Mech. 16 (1967), 1361-1376.

California Institute of Technology 